International Mathematical Forum, 2, 2007, no. 58, 2875 - 2888

\title{
On the Existence of a Fixed Point for Mutually Contractive Self-Mappings in Intuitionistic Generalized Fuzzy Metric Spaces
}

\author{
Renu Chugh and Seema Mehra \\ Department of Mathematics \\ M.D. University, Rohtak - 124 001, India \\ chughrenu@yahoo.com \\ smehra2007@yahoo.co.in
}

\begin{abstract}
The purpose of this paper is to define a sequence of mutually contractive self-mappings in intuitionistic generalized fuzzy metric spaces as defined by authors in [16] and prove a common fixed point theorem for such mappings in this newly defined space. Our result generalizes and extends many known results in generalized fuzzy metric spaces, fuzzy metric spaces and metric spaces.
\end{abstract}

Mathematics Subject Classification: 54H25, 54E40, 54E35

Keywords: Generalized Fuzzy Metric Spaces, Intuitionistic Fuzzy Metric Spaces, Intuitionistic Generalized Fuzzy Metric Spaces, mutually maps, intuitionistic mutually maps, fixed points

\section{Introduction}

Zadeh [11] introduced the concept of fuzzy set. After that a lot of work have been done regarding fuzzy sets. Fuzzy metric spaces have been introduced and studied in a number of ways. Amongst the various ways of introducing fuzzy metric spaces, one is by generalizing the concept of Probabilistic metric spaces. Probabilistic metric spaces [2] have been studied extensively in recent times, especially fixed point studies on such spaces have been a very attractive problem of mathematics. In 1975, Kramosil and Michalek [13] introduced the 
fuzzy metric space by generalizing the concept of probabilistic metric space to the fuzzy situation. Grabiec [12] proved the contraction principle in the setting of fuzzy metric spaces introduced by Kramosil and Michalek [13]. Grabiec's [12] results were further generalized by Subrahmanyam [14] for a pair of commuting maps. Motivated by the measure of nearness between two or more objects with respect to a specific property, called the parameter of the nearness, Dhage, in 1984 in his Ph.D. thesis [5] introduced the theory of D-metric spaces by which it has been possible to determine the distance between two or more points of the set under consideration. For details on D-metric space see Dhage $[4,6]$. In 2002, Chugh and Kumar [15] suggested a generalization of fuzzy metric space by introducing the concept of generalized fuzzy metric space in the following way:

Definition $1.1([\mathbf{1 5}])$. The triplet $(X, S, *)$ is an $S$-fuzzy metric space if $X$ is an arbitrary set, $*$ is a continuous $t$-norm and $S$ is a fuzzy set in $X^{3} \times[0, \infty)$ satisfying the following conditions:

(1) $S(x, y, z, 0)=0$

(2) $S(x, y, z, t)=1$ for all $t>0$ if and only if $x=y=z$ (coincidence)

(3) $S(x, y, z, t)=S(y, z, x, t)=S(z, y, x, t) \ldots$ (symmetry)

(4) $S(x, y, z, r+s+t) \geq S(x, y, w, r) * S(x, w, z, s) * S(w, y, z, t)$ and $r, s, t>0$ (rectangle or tetrahedral inequality)

(5) $S(x, y, z, \cdot):[0, \infty) \rightarrow[0,1]$ is left continuous

(6) $\lim _{t \rightarrow \infty} S(x, y, z, t)=1$ for all $x, y, z$ in $X$.

Where the mapping $*:[0,1]^{3} \rightarrow[0,1]$ is called $t$-norm if

$$
\begin{aligned}
& (T-1) *(a, 1,1)=a \text { for all } a \in[0,1] \\
& (T-2) *(a, b, c)=*(a, c, b)=*(c, a, b) \ldots, \\
& (T-3) *(a, b, c) \leq *(d, e, f), \text { for } d \geq a, e \geq b, f \geq c, \\
& (T-4) *(*(a, b, c), d, e)=*(a, *(b, c, d), e)=*(a, b, *(c, d, e))
\end{aligned}
$$

Examples of such $t$-norm is $a * b * c=a b c$.

As a generalization of fuzzy sets, Atanassov [10] introduced and studied the concept of intuitionistic fuzzy sets. Recently, using the idea of intuitionistic fuzzy sets, Park [9] introduced the notion of intuitionistic fuzzy metric spaces with the help of continuous $t$-norms and continuous $t$-conorms as a generalization of fuzzy metric spaces due to George and Veeramani [1], and showed that every metric induces on intuitionistic fuzzy metric, every fuzzy metric space 
is an intuitionistic fuzzy metric space and found a necessary and sufficient condition for an intuitionistic fuzzy metric space to be complete.

Choudhary [7] introduced mutually contractive sequence of self maps and proved a fixed point theorem. Servet Kutukcu [17] obtained a common fixed point theorem for a sequence of self maps in intuitionistic fuzzy metric spaces. The purpose of this paper, using the idea of intuitionistic fuzzy set due to Atanassov [10], we define the notion of intuitionistic generalized fuzzy metric spaces due to Kramosil and Michalek [13], Chugh and Sanjay [15], and Turkoglu, Alaca and Yildiz [8], is to obtain a common fixed point theorem by using a new contractive condition in intuitionistic generalized fuzzy metric spaces. Our result generalizes and extends many known results in generalized fuzzy metric spaces, fuzzy metric spaces and metric spaces.

We introduce the concept of generalized intuitionistic fuzzy metric space as follows:

Definition 1.2. The mapping $*:[0,1]^{3} \rightarrow[0,1]$ is a continuous $t$-norm if $*$ satisfies the following conditions:

(a) $*(a, 1,1)=a$ for all $a \in[0,1]$,

(b) $*(a, b, c)=*(a, c, b)=*(c, a, b) \ldots$,

(c) $*(a, b, c) \leq *(d, e, f)$, for $d \geq a, e \geq b, f \geq c$,

(d) $*(*(a, b, c), d, e)=*(a, *(b, c, d), e)=*(a, b, *(c, d, e))$

Definition 1.3. The mapping $\diamond:[0,1]^{3} \rightarrow[0,1]$ is a continuous $t$-conorm if satisfies the following conditions:

(a) $\diamond(a, 0,0)=a$ for all $a \in[0,1]$,

(b) $\diamond(a, b, c)=\diamond(a, c, b)=\diamond(c, a, b) \ldots$,

$(\mathrm{c}) \diamond(\diamond(a, b, c) d, e)=\diamond(a, \diamond(b, c, d), e)=\diamond(a, b, \diamond(c, d, e))$,

$(\mathrm{d}) \diamond(a, b, c) \leq \diamond(d, e, f)$, for $d \geq a, e \geq b, f \geq c$ and $a, b, c, d, e, f \in[0,1]$

Definition 1.4. A 5-tuple $(X, M, N, *, \diamond)$ is called a intuitionistic generalized fuzzy metric space if $X$ is an arbitrary nonempty set, $*$ is a continuous $t$-norm, $\diamond$ is a continuous $t$-conorm and $M, N$ are fuzzy sets on $X^{3} \times[0, \infty)$ satisfying the following conditions for each $x, y, z \in X$ and $r, s, t>0$

(a) $M(x, y, z, t)+N(x, y, z, t) \leq 1$ for all $x, y, z \in X$ and $t>0$;

(b) $M(x, y, z, 0)=0$ for all $x, y, z \in X$;

(c) $M(x, y, z, t)=1$ for all $x, y, z \in X$ and $t>0$ iff $x=y=z$; 
(d) $M(x, y, z, t)=M(y, z, x, t)=M(z, y, x, t) \ldots$ for all $x, y, z \in X$ and $t>0$

(e) $M(x, y, w, r) * M(w, y, z, s) * M(x, w, z, t) \leq M(x, y, z, r+s+t)$ for all $x, y, z \in X$ and $r, s, t>0$;

(f) for all $x, y, z \in X, M(x, y, z, \cdot):[0, \infty) \rightarrow[0,1]$ is left continuous;

(g) $\lim _{t \rightarrow \infty} M(x, y, z, t)=1$ for all $x, y, z \in X$ and $t>0$;

(h) $N(x, y, z, 0)=1$ for all $x, y, z \in X$;

(i) $N(x, y, z, t)=0$ for all $x, y, z \in X$ and $t>0$ if and only if $x=y=z$;

(j) $N(x, y, z, t)=N(y, z, x, t)=N(z, y, x, t) \ldots$ for all $x, y, z \in X$ and $t>0$

(k) $N(x, y, w, r) \diamond N(w, y, z, s) \diamond N(x, w, z, t) \geq N(x, y, z, r+s+t)$ for all $x, y, z \in X$ and $r, s, t>0$;

(l) for all $x, y, z \in X, N(x, y, z, \cdot):[0, \infty) \rightarrow[0,1]$ is right continuous.

(m) $\lim _{t \rightarrow \infty} N(x, y, z, t)=0$ for all $x, y, z \in X$ and $t>0$;

Then $(M, N)$ is called an intuitionistic generalized fuzzy metric on $X$. The functions $M(x, y, z, t)$ and $N(x, y, z, t)$ denote the degree of parameter of nearness and the degree of parameter of non-nearness among $x, y$ and $z$ with respect to $t$, respectively.

Example 1. Let $X=\mathbb{R}$ and $M, N$ be fuzzy sets on $X^{3} \times[0, \infty)$ defined by

$$
M(x, y, z, t)=\min \left\{M_{d}(x, y, t), M_{d}(y, z, t), M_{d}(x, z, t)\right\}
$$

and

$$
N(x, y, z, t)=\max \left\{N_{d}(x, y, t), N_{d}(y, z, t), N_{d}(x, z, t)\right\}
$$

for all $x, y, z$ in $X, t>0 ; M_{d}(x, y, t)$ and $N_{d}(x, y, t)$ are fuzzy sets defined as

$$
M_{d}(x, y, t)=\frac{t}{t+|x-y|} \quad \text { and } \quad N_{d}=\frac{|x-y|}{t+|x-y|} .
$$

Now we show that $(X, M, N, *, \diamond)$ is intuitionistic generalized fuzzy metric space.

Here $*=\min$ and $\diamond=\max$.

(i) for all $x, y, z$ in $X$ and $t=0$, we have $t /(t+|x-y|)=0$ which implies,

$$
M(x, y, z, t)=\min \left\{M_{d}(x, y, t), M_{d}(y, z, t), M_{d}(x, z, t)\right\}=0
$$


(ii) $M(x, y, z, t)=1$

$$
\begin{array}{ll}
\Leftrightarrow & \min \left\{M_{d}(x, y, t), M_{d}(y, z, t), M_{d}(x, z, t)\right\}=1 \\
\Leftrightarrow & M_{d}(x, y, t)=M_{d}(y, z, t)=M_{d}(x, z, t)=1 \\
\Leftrightarrow & x=y=z
\end{array}
$$

(iii) It can be obviously seen that

$$
M(x, y, z, t)=\{M(y, z, x, t)=M(z, x, y, t) \ldots
$$

(iv) $M(x, y, z, r+s+t)$

$$
\begin{aligned}
& =\min \left\{M_{d}(x, y, r+s+t), M_{d}(y, z, r+s+t), M_{d}(x, z, r+s+t)\right\} \\
& M(x, y, z, r+s+t) \\
& =\min \left\{\frac{r+s+t}{r+s+t+|x-y|}, \frac{r+s+t}{r+s+t+|y-z|}, \frac{r+s+t}{(r+s+t)+|x-z|}\right\} \\
& (r+s+t)+|x-y| \geq r+|x-y| \text { and so } \\
& \frac{r+s+t}{r+s+t+|x-y|} \geq \frac{r}{r+|x-y|} \\
& M(x, y, z, r+s+t) \geq \min \left\{\frac{r}{r+|x-y|}, \frac{s}{s+|y-z|}, \frac{t}{t+|x-z|}\right\} \\
& \geq \min \left\{\min \left\{\frac{r}{r+|x-y|}, \frac{r}{r+|y-w|}, \frac{r}{r+|x-w|}\right\},\right. \\
& \min \left\{\frac{s}{s+|w-y|}, \frac{s}{s+|y-z|}, \frac{s}{s+|z-w|}\right\} \text {, } \\
& \left.\min \left\{\frac{t}{t+|x-w|}, \frac{t}{t+|z-w|}, \frac{t}{t+|x-z|}\right\}\right\} \\
& =\min \left\{\min \left\{M_{d}(x, y, r), M_{d}(y, w, r), M_{d}(x, w, r)\right\}\right. \\
& \min \left\{M_{d}(y, z, s), M_{d}(y, w, s), M_{d}(z, w, s)\right\} \\
& \left.\min \left\{M_{d}(x, w, t), M_{d}(z, w, t), M_{d}(x, z, t)\right\}\right\} \\
& =\min \{M(x, y, w, r), M(y, z, w, s), M(x, z, w, t)\} \\
& =M(x, y, w, r) * M(w, y, z, s) * M(x, w, z, t) \\
& M(x, y, z, r+s+t) \geq M(x, y, w, r) * M(w, y, z, s) * M(x, w, z, t)
\end{aligned}
$$

(v) $M(x, y, z, \cdot):[0, \infty) \rightarrow[0,1]$ is left continuous for $x, y, z \in X$ and $r, s, t>$ 0 .

(vi) $\lim _{t \rightarrow \infty} M(x, y, z, t)=1$ for all $x, y, z$ in $X$.

Similarly 
(i) for all $x, y, z$ in $X$ and $t=0$, we have $|x-y| /(t+|x-y|)=1$

which implies, $N(x, y, z, t)=\max \left\{N_{d}(x, y, t), N_{d}(y, z, t), N_{d}(x, z, t)\right\}=1$

(ii) $N(x, y, z, t)=0$

$\Leftrightarrow \quad \max \left\{N_{d}(x, y, t), N_{d}(y, z, t), N_{d}(x, z, t)\right\}=0$

$\Leftrightarrow \quad N_{d}(x, y, t)=N_{d}(y, z, t)=N_{d}(x, z, t)=0$

$\Leftrightarrow \quad x=y=z$

(iii) It can be obviously seen that

$N(x, y, z, t)=N(y, z, x, t)=N(z, x, y, t) \ldots$

(iv) $N(x, y, z, r+s+t)$

$$
\begin{aligned}
& =\max \left\{N_{d}(x, y, r+s+t), N_{d}(y, z, r+s+t), N_{d}(z, x, r+s+t)\right\} \\
& =\max \left\{\frac{|x-y|}{r+s+t+|x-y|}, \frac{|y-z|}{r+s+t+|y-z|}, \frac{|x-z|}{r+s+t+|x-z|}\right\} \\
& r+s+t \geq r \\
& r+s+t+|x-y| \geq r+|x-y| \\
& \frac{1}{r+s+t+|x-y|} \leq \frac{1}{r+|x-y|} \\
& \frac{|x-y|}{r+s+t+|x-y|} \leq \frac{|x-y|}{r+|x-y|} \\
& \leq \max \left\{\frac{|x-y|}{r+|x-y|}, \frac{|y-z|}{s+|y-z|}, \frac{|x-z|}{t+|x-z|}\right\} \\
& \leq \max \left\{\max \left\{\frac{|x-y|}{r+|x-y|}, \frac{|y-w|}{r+|y-w|}, \frac{|x-w|}{r+|x-w|}\right\},\right. \\
& \max \left\{\frac{|y-z|}{s+|y-z|}, \frac{|w-y|}{s+|w-y|}, \frac{|z-w|}{s+|z-w|}\right\} \text {, } \\
& \left.\max \left\{\frac{|x-w|}{t+|x-w|}, \frac{|z-w|}{t+|z-w|}, \frac{|x-z|}{t+|x-z|}\right\}\right\} \\
& =\max \left\{\max \left\{N_{d}(x, y, r), N_{d}(y, w, r), N_{d}(x, w, r)\right\}\right. \\
& \max \left\{N_{d}(y, z, s), N_{d}(y, w, s), N_{d}(z, w, s)\right\} \\
& \left.\max \left\{N_{d}(x, w, t), N_{d}(z, w, t), N_{d}(x, z, t)\right\}\right\} \\
& =\max \{N(x, y, w, r), N(y, z, w, s), N(x, z, w, t)\} \\
& =N(x, y, w, r) \diamond N(y, z, w, s) \diamond N(x, w, z, t)
\end{aligned}
$$

(v) $N(x, y, z, \cdot):[0, \infty) \rightarrow[0,1]$ is right continuous.

(vi) $\lim _{t \rightarrow \infty} N(x, y, z, t)=0$ for all $x, y, z$ in $X$. 
Thus $(X, M, N, *, \diamond)$ is generalized Intuitionistic fuzzy metric space.

Definition 1.5. Let $(X, M, N, *, \diamond)$ be an intuitionistic generalized fuzzy metric space. Then

(a) a sequence $\left\{x_{n}\right\}$ in $X$ is convergent to $x \in X$ if, for all $t>0$

$$
\lim _{n \rightarrow \infty} M\left(x_{n}, x_{n+p}, x, t\right)=1 \text { and } \lim _{n \rightarrow \infty} N\left(x_{n}, x_{n+p}, x, t\right)=0
$$

(b) a sequence $\left\{x_{n}\right\}$ in $X$ is Cauchy if for all $t>0$ and $p, q>0$

$$
\lim _{n \rightarrow \infty} M\left(x_{n}, x_{n+p}, x_{n+p+q}, t\right)=1 \quad \text { and } \quad \lim _{n \rightarrow \infty} N\left(x_{n}, x_{n+p}, x_{n+p+q}, t\right)=0
$$

(c) A intuitionistic generalized fuzzy metric space in which every cauchy sequence is convergent is said to be complete.

Definition $1.6([\mathbf{1 7}])$. A sequence $\left\{T_{i}\right\}$ of self maps on a complete intuitionistic fuzzy metric space $(X, M, N, *, \diamond)$ is said to intuitionistic mutually contractive if for $t>0$ and $i \in \mathbf{N}$.

$$
M\left(T_{i} x, T_{j} y, t\right) \geq M(x, y, t / k)
$$

and

$$
N\left(T_{i} x, T_{j} y, t\right) \leq N(x, y, t / k)
$$

where $x, y \in X, k \in(0,1), i \neq j$ and $x \neq y$.

Definition 1.7. A sequence $\left\{T_{i}\right\}$ of self maps on complete intuitionistic generalized fuzzy metric space $(X, M, N, *, \diamond)$ is said to be intuitionistic mutually contractive for $t>0$ and $i \in N$

$$
\begin{aligned}
M\left(T_{i} x, T_{j} y, T_{\ell}, t\right) & \geq M(x, y, z, t / k) \\
N\left(T_{i} x, T_{j} y, T_{\ell}, t\right) & \leq N(x, y, z, t / k)
\end{aligned}
$$

where $x, y, z \in X, k \in(0,1), i \neq j \neq \ell$ and $x \neq y \neq z$. 


\section{Main Results}

Theorem 1. Let $(X, M, N, *, \diamond)$ be a complete intuitionistic generalized fuzzy metric space and $\left\{T_{n}\right\}$ be a sequence of self maps of $X$ satisfying

(a) $T_{i} T_{j}=T_{j} T_{i}$ for all $i, j=1,2, \ldots$

(b) $T_{i}$ is continuous for all $i=1,2, \ldots$.

(c) $\left\{T_{i}\right\}$ is intuitionistic mutually contractive.

Then $\left\{T_{i}\right\}$ has a unique common fixed point.

Proof. Let $x_{0}$ be any point in $X$. We can construct a sequence $\left\{x_{n}\right\}$ in $X$ such that

$$
x_{1}=T_{1} x_{0}, x_{2}=T_{2} x_{1}, \ldots, x_{n}=T_{n} x_{n-1}, \ldots
$$

Case I. If no terms of $\left\{x_{n}\right\}$ are equal. Then for $t>0$ using (c), we get

$$
\begin{aligned}
M\left(x_{n}, x_{n+1}, x_{n+p}, t\right) & =M\left(T_{n} x_{n-}, T_{n+1} x_{n}, T_{n+p} x_{n+p-1}, t\right) \\
& \geq M\left(x_{n-1}, x_{n}, x_{n+p-1}, t / k\right) \\
N\left(x_{n}, x_{n+1}, x_{n+p}, t\right) & =N\left(T_{n} x_{n-1}, T_{n+1} x_{n}, T_{n+p} x_{n+p-1}, t\right) \\
& \leq N\left(x_{n-1}, x_{n}, x_{n+p-1}, t / k\right)
\end{aligned}
$$

By repeated application of above inequalities, we get:

$$
\begin{aligned}
M\left(x_{n}, x_{n+1}, x_{n+p}, t\right) & \geq M\left(x_{0}, x_{1}, x_{p}, t / k^{n}\right) \\
N\left(x_{n}, x_{n+1}, x_{n+p}, t\right) & \leq N\left(x_{0}, x_{1}, x_{p}, t / k^{n}\right)
\end{aligned}
$$

Then, using (e) and (k) of definition 1.4, we get:

Let $t>0$ be arbitrary. We define $t_{1}=t / 2 p-1$

$$
\begin{aligned}
& M\left(x_{n}, x_{n+p}, x_{n+p+q}, t\right) \\
& \geq \quad M\left(x_{n}, x_{n+1}, x_{n+p+q}, t_{1}\right) * M\left(x_{n}, x_{n+1}, x_{n+p}, t_{1}\right) \\
& \quad * M\left(x_{n+1}, x_{n+p}, x_{n+p+q}, t-2 t_{1}\right)
\end{aligned}
$$




$$
\begin{aligned}
\geq & M\left(x_{0}, x_{1}, x_{p+q}, t_{1} / k^{n}\right) * M\left(x_{0}, x_{1}, x_{p}, t_{1} / k^{n}\right) \\
& M\left(x_{n+1}, x_{n+p}, x_{(n+1)+(p-1)+q}, t-2 t_{1}\right) \\
\geq & \left.M x_{0}, x_{1}, x_{p+q}, t_{1} / k^{n}\right) * M\left(x_{0}, x_{1}, x_{p}, t_{1} / k^{n}\right) \\
& * M\left(x_{n+1}, x_{n+2}, x_{(n+1)+(p-)+q}, t_{1}\right) \\
& * M\left(x_{n+1}, x_{(n+1)+(p-1)}, x_{n+2}, t_{1}\right) \\
& * M\left(x_{n+2}, x_{(n+2)+(p-2)}, x_{(n+2)+(p-2)+q}, t-4 t_{1}\right) \\
\geq & M\left(x_{0}, x_{1}, x_{p+q}, t_{1} / k^{n}\right) * M\left(x_{0}, x_{1}, x_{p}, t_{1} / k^{n}\right) \\
& * M\left(x_{0}, x_{1}, x_{p+q-1}, t_{1} / k^{n+1}\right) * M\left(x_{0}, x_{1}, x_{p-1}, t_{1} / k^{n+1}\right) \\
& * M\left(x_{n+2}, x_{(n+2)+(p-2)}, x_{(n+2)+(p-2)+q}, t-4 t_{1}\right)
\end{aligned}
$$

and

$$
\begin{aligned}
& N\left(x_{n}, x_{n+p}, x_{n+p+q}, t\right) \\
& \leq \quad \\
& N\left(x_{n}, x_{n+1}, x_{n+p+q}, t_{1}\right) \diamond N\left(x_{n}, x_{n+1}, x_{n+p}, t_{1}\right) \\
& \leq N\left(x_{0}, x_{1}, x_{p+q}, t_{1} / k^{n}\right) \diamond N\left(x_{0}, x_{1}, x_{p}, t_{1} / k^{n}\right) \\
& \diamond N\left(x_{n+1}, x_{n+p}, x_{(n+1)+(p-1)+q}, t-2 t_{1}\right) \\
& \leq N\left(x_{0}, x_{1}, x_{p+q}, t_{1} / k^{n}\right) \diamond N\left(x_{0}, x_{1}, x_{p} t_{1} / k^{n}\right) \\
& \diamond N\left(x_{n+1}, x_{n+2}, x_{(n+1)+(p-1)+q}, t_{1}\right) \diamond N\left(x_{n+1}, x_{(n+1)+(p-1)}, x_{n+2}, t_{1}\right) \\
& \diamond N\left(x_{n+2}, x_{(n+2)+(p-2)}, x_{(n+2)+(p-2)+q}, t-4 t_{1}\right) \\
& \leq N\left(x_{0}, x_{1}, x_{p+q}, t_{1} / k^{n}\right) \diamond N\left(x_{0}, x_{1}, x_{p}, t_{1} / k^{n}\right) \\
& \diamond N\left(x_{0}, x_{1}, x_{p+q-1}, t_{1} / k^{n+1}\right) \diamond N\left(x_{0}, x_{1}, x_{p-1}, t_{1} / k^{n+1}\right) \\
& \diamond N\left(x_{n+2}, x_{(n+2)}, x_{(n+2)+(p-2)}, x_{(n+2)+(p-2)+q}, t-4 t_{1}\right)
\end{aligned}
$$

Repeating the above procedure $(p-1)$ times we obtain, For all $t>0$.

$$
\begin{aligned}
& M\left(x_{n}, x_{n+p}, x_{n+p+q}, t\right) \\
& \geq\left(x_{0}, x_{1}, x_{p+q}, t_{1} / k^{n}\right) * M\left(x_{0}, x_{1}, x_{p}, t_{1} / k^{n}\right) \\
& * M\left(x_{0}, x_{1}, x_{p+q-1}, t_{1} / k^{n+1}\right) * M\left(x_{0}, x_{1}, x_{p-1}, t_{1} / k^{n+1}\right) \ldots \\
& * M\left(x_{0}, x_{1}, x_{2}, t_{1} / k^{n+p-2}\right) * M\left(x_{n+p-1}, x_{n+p}, x_{n+p+q}, t_{1}\right) \\
& \geq M\left(x_{0}, x_{1}, x_{p+q}, t_{1} / k^{n}\right) * M\left(x_{0}, x_{1}, x_{p}, t_{1} / k^{n}\right) \\
& * M\left(x_{0}, x_{1}, x_{p+q-1}, t_{1} / k^{n+1}\right) * M\left(x_{0}, x_{1}, x_{p-1}, t_{1} / k^{n+1}\right) \ldots \\
& * M\left(x_{0}, x_{1}, x_{2}, t_{1} / k^{n+p-2}\right) * M\left(x_{0}, x_{1}, x_{q+1}, t_{1} / k^{n+p-1}\right)
\end{aligned}
$$


and

$$
\begin{aligned}
& N\left(x_{n}, x_{n+p}, x_{n+p+q}, t\right) \\
& \leq \quad N\left(x_{0}, x_{1}, x_{p+q}, t_{1} / k^{n}\right) \diamond N\left(x_{0}, x_{1}, x_{p}, t_{1} / k^{n}\right) \\
& \diamond N\left(x_{0}, x_{1}, x_{p+q-1}, t_{1} / k^{n+1}\right) \diamond N\left(x_{0}, x_{1}, x_{p-1}, t_{1} / k^{n+1}\right) \ldots \\
& \diamond N\left(x_{0}, x_{1}, x_{2}, t_{1} / k^{n+p-1}\right) \diamond N\left(x_{n+p-1}, x_{n+p}, x_{n+p+q}, t_{1}\right) \\
& \leq N\left(x_{0}, x_{1}, x_{p+q}, t_{1} / k^{n}\right) \diamond N\left(x_{0}, x_{1}, x_{p}, t_{1} / k^{n}\right) \\
& \diamond N\left(x_{0}, x_{1}, x_{p+q-1}, t_{1} / k^{n+1}\right) \diamond N\left(x_{0}, x_{1}, x_{p-1}, t_{1} / k^{n+1}\right) \ldots \\
& \diamond N\left(x_{0}, x_{1}, x_{2}, t_{1} / k^{n+p-2}\right) \diamond N\left(x_{0}, x_{1}, x_{q+1}, t_{1} / k^{n+p-1}\right)
\end{aligned}
$$

According to $(\mathrm{g})$ and $(\mathrm{m})$ of definition 1.4 , we now get:

$$
\lim M\left(x_{n}, x_{n+p}, x_{n+p+q}, t\right) \geq 1 * 1 * 1 * \ldots * 1
$$

and

$$
\lim N\left(x_{n}, x_{n+p}, x_{n+p+q}, t\right) \leq 0 \diamond 0 \diamond 0 \diamond \ldots \diamond 0
$$

which implies that

$$
M\left(x_{n}, x_{n+p}, x_{n+p+q}, t\right) \rightarrow 1
$$

and

$$
N\left(x_{n}, x_{n+p}, x_{n+p+q}, t\right) \rightarrow 0 .
$$

That is, $\left\{x_{n}\right\}$ is a cauchy sequence in $X$, hence convergent. Call the limit $z$.

Since two consecutive terms of $\left\{x_{n}\right\}$ are unequal, there exists an increasing sequence $\{n(r)\}$ of positive integers such that $x_{n(r)-1} \neq z$. For arbitrary $t>0$ and positive integer $j$, we have for any $n(r)>j$ and any positive integer $p$,

$$
\begin{aligned}
M\left(x_{n(r)}, x_{n(r)+p}, T_{j} z, t\right) & =M\left(T_{n(r)} x_{n(r)-1}, T_{n(r)+p} x_{n(r)+p-1}, T_{j} z, t\right) \\
& \geq M\left(x_{n(r)-1}, x_{n(r)-1+p}, z, t\right) \\
& \rightarrow 1 \text { as } r \rightarrow \infty
\end{aligned}
$$

and

$$
\begin{aligned}
N\left(x_{n(r)}, x_{n(r)+p}, T_{j} z, t\right) & =N\left(T_{n(r)} x_{n(r)-1}, T_{n(r)+p} x_{n(r)+p-1}, T_{j} z, t\right) \\
& \leq N\left(x_{n(r)-1}, x_{n(r)-1+p}, z, t\right) \\
& \rightarrow 0 \text { as } r \rightarrow \infty
\end{aligned}
$$

Therefore $\left\{x_{n(r)}\right\} \rightarrow T_{j} z$ as $r \rightarrow \infty$ that is, $z=T_{j} z$ for all $j=1,2, \ldots$

Case II. $x=T_{i} x$ for some $i$ 
That is, for some $i$, there exists $z \in X$ such that

$$
z=T_{i} z
$$

If possible, let $z \neq T_{j} z$ for some $j$. Then the following two sub-cases may arise.

Subcase IIA.

$$
z \neq T_{j}^{n} z \text { for all } n=1,2, \ldots
$$

Then for any positive integer $n$, and $t>0$

$$
\begin{aligned}
M\left(z, T_{j}^{n+p} z, T_{j}^{n} z, t\right) & =M\left(T_{j} z, T_{j}\left(T_{j}^{n+p-1} z\right), T_{j}\left(T_{j}^{n+p-1} z\right), t\right) \\
& \geq M\left(z, T_{j}^{n+p-1} z, T_{j}^{n-1} z, t / k\right)
\end{aligned}
$$

and

$$
\begin{aligned}
N\left(z, T_{j}^{n+p} z, T_{j}^{n} z, t\right) & =N\left(T_{j} z, T_{j}\left(T_{j}^{n+p-1} z\right), T_{j}\left(T_{j}^{n-1} z\right), t\right) \\
& \leq N\left(z, T_{j}^{n+p-1} z, T_{j}^{n-1} z, t / k\right) .
\end{aligned}
$$

Consequently,

$$
M\left(z, T_{j}^{n+p} z, T_{j}^{n} z, t\right) \geq\left(z, T_{j}^{p} z, T_{j} z, t / k^{n}\right) \rightarrow 1 \text { as } n \rightarrow \infty
$$

and

$$
N\left(z, T_{j}^{n+p} z, T_{j}^{n} z, t\right) \leq\left(z, T_{j}^{p} z, T_{j} z, t / k^{n}\right) \rightarrow 0 \text { as } n \rightarrow \infty
$$

we get

$$
T_{j}^{n} z \rightarrow z \quad \text { as } n \rightarrow \infty
$$

Since $T_{j}$ is continuous, we get:

$$
T_{j}^{n} z=T_{j}\left(T_{j}^{n-1} z\right) \rightarrow T_{j} z \text { as } n \rightarrow \infty
$$

This shows that $T_{j} z=z$ which is a contradiction.

Hence $z=T_{j}^{r} z$ for some $r$.

Subcase IIB. Let $z=T_{j}^{n} z$ for some positive integer $n$.

Let $r$ be the smallest integer with this property. Then, we get:

$$
z \neq T_{j}^{m} z \text { for some } m=1,2, \ldots, r-1
$$

and for $t>0$

$$
\begin{aligned}
M\left(z, z, T_{j} z, t\right) & =M\left(T_{i} z, T_{j}^{r} z, T_{j}\left(T_{i} z\right), t\right) \\
& \geq\left(T_{i} z, T_{j}\left(T_{j}^{n-1} z\right), T_{i}\left(T_{j} z\right), t\right) \quad\left(\text { since } T_{i} T_{j}=T_{j} T_{i}\right)
\end{aligned}
$$




$$
\begin{aligned}
& \geq\left(z, T_{j}^{n-1} z, T_{j} z, t / k\right) \\
& \geq \cdots \cdots \\
& \geq\left(z, T_{j}^{2} z, T_{j} z, t / k^{r-2}\right) \\
& =M\left(T_{i} z, T_{j}^{2}\left(T_{i} z\right), T_{j} z, t / k^{r-2}\right) \\
& =M\left(T_{i} z, T_{i}\left(T_{j}^{2} z\right), T_{j} z, t / k^{r-2}\right) \\
& \geq M\left(z, T_{j}^{2} z, z, t / k^{r-1}\right) \quad\left(\text { Since } T_{j}^{2} z \neq z\right) \\
& \geq M\left(T_{i} z, T_{j}\left(T_{j} z\right), T_{i} z, t / k^{r-1}\right) \\
& \geq M\left(z, T_{j} z, z, t / k^{r}\right)
\end{aligned}
$$

and

$$
\begin{aligned}
N\left(z, z, t_{j} z, t\right) & =N\left(T_{i} z, T_{j}^{r} z, T_{j}\left(T_{i} z\right), t\right) \\
& \left.\geq N\left(T_{i} z, T_{j}\left(T_{j}^{n-1} z\right), T_{i}\left(T_{j} z\right), t\right) \quad \text { Since } T_{i} T_{j}=T_{j} T_{i}\right) \\
& \leq N\left(z, T_{j}^{n-1} z, T_{j} z, t / k\right) \\
& \leq \cdots \cdots \\
& \leq N\left(z, T_{j}^{2} z, T_{j} z, t / k^{r-2}\right) \\
& =N\left(T_{i} z, T_{j}^{2}\left(T_{i} z\right), T_{j} z, t / k^{r-2}\right) \\
& =N\left(T_{i} z, T_{i}\left(T_{j}^{2} z\right), T_{j} z, t / k^{r-2}\right) \\
& \leq N\left(z, T_{j}^{2} z, z, t / k^{r-1}\right) \quad\left(\text { Since } T_{j}^{2} z \neq z\right) \\
& \leq N\left(T_{i} z, T_{j}\left(T_{j} z\right), T_{i} z, t / k^{r-1}\right) \\
& \leq N\left(z, T_{j} z, z, t / k^{r}\right)
\end{aligned}
$$

But this gives a contradiction, so $z=T_{j} z$ for all $j=1,2, \ldots$, .

To show uniqueness, assume $z$ and $w$ be two common fixed points such that $z \neq w$. Then, using $(\mathrm{c})$, we get

$$
M(z, z, w, t)=M\left(T_{i} z, T_{j} z, T_{\ell}, t\right) \geq(z, z, w, t / k)
$$

and

$$
N(z, z, w, t)=N\left(T_{i} z, T_{j} z, T_{\ell}, t\right) \leq(z, z, w, t / k) .
$$

Which is a contradiction. Therefore, $z=w$. Hence the common fixed point is unique.

\section{References}

[1] A. George and P. Veeramani, On some results in fuzzy metric spaces, Fuzzy Sets and Systems, 64(1994), 395-399. 
[2] B. Schweizer and A. Sklar, Probabilistic Metric Spaces, North Holland Amsterdam (1983).

[3] B. Singh and M.S. Chauhan, Generalized fuzzy metric space and fixed point theorems, Bull. Cal. Math. Soc., 89(1997), 457-460.

[4] B.C. Dhage, A.M. Pathan and B.E. Rhoades, A general existence principle for fixed point theorems in D-metric spaces. Internat. Journal Math. and Math. Sci., 23(2000), 441-448.

[5] B.C. Dhage, A study of some fixed point theorems, Ph.D. Thesis, Marathwada University, Aurangabad (1984), India.

[6] B.C. Dhage, On common fixed point of coincidentally commuting mappings in D-metric spaces, Indian J. Pure Appl. Math., 30(3) (1999), 395406.

[7] B.S. Choudhary, A unique common fixed point theorem for a sequence of self-mapping in Menger spaces, Bull. Korean Math. Soc.,37(3)(2000), 569-575.

[8] C. Alaca, D. Turkoglu and C. Yildiz, Fixed points in intuitionistic fuzzy metric spaces, Choas, Solitions and Fractals, 29(2006), 1073-1078.

[9] J.H. Park, Intuitionistic fuzzy metric spaces, Chaos, Solitons and Fractals, 22(2004), 1039-1046.

[10] K. Atanassov, Intuitionistic fuzzy sets, Fuzzy Sets and Systems, 20(1986), $87-96$.

[11] L.A. Zadeh, Infor. Control, 89(1965), 338.

[12] M. Grabiec, Fixed points in Fuzzy metric spaces, Fuzzy sets and systems, 27(1983), 385-389.

[13] O. Kramsoil and J. Michalek, Fuzzy metric and statistical metric spaces, Kybernetica, 11(1975), 330-334.

[14] P.V. Subrahmanyam, A common fixed point theorem in fuzzy metric spaces, Information Sci., 83(1995), 109-112.

[15] R. Chugh and S. Kumar, Weakly compatible maps in generalized fuzzy metric space, Journal of Analysis, 10(2002), 65-74. 
[16] R. Chugh and S. Mehra, Fixed points in intuitionistic generalized fuzzy metric spaces, communicated.

[17] S. Kutukcu, A common fixed point theorem for a sequence of self maps in intuitionistic fuzzy metric spaces, Commun. Korean Math. Soc., $\mathbf{2 1}(4)(2006), 679-687$.

Received: May 17, 2007 